\title{
Marcação Diferencial do Objeto direto (DOM) em Kimwani, uma língua bantu
}

\author{
Calawia Salimo* \\ Marco Antonio Rocha Martins*
}

Resumo: Descrevemos e analisamos o padrão de marcação dos objetos diretos em Kimwani. Especificamente descrevemos a tipologia de DOM e apresentamos evidências de que a DOM em Kimwani não está relacionada com a escala ou o grau de afetação, mas, sim, pela hierarquia de relevância do objeto: os traços [+humano] e [+animalidade] estão no ponto mais alto da hierarquia, e requerem marcação diferencial na posição de objeto direto. Esse resultado aponta para uma direção contrária da proposta teórica de Dowty (1991) com base em dados do inglês, Hopper e Thompson (1980) e Næss (2004) com recurso a dados de várias línguas.

Palavras-chave: Marcação do Objeto direto. Afetação. Língua bantu. Kimwani.

Abstract: We describe and analyze the marking pattern of direct objects in Kimwani. We describe the Marking Type of the DOM and we present evidence that, in Kimwani the relevance hierarchy of the object does not relate the DOM to the scale or degree of affectation, but rather the [+ human] and [+ animality] traits are at the highest point of the hierarchy, and require differential marking in the direct object position. This result points in the opposite direction from Dowty (1991) theoretical proposal based on data from English, Hopper and Thompson (1980) and Næss (2004) using data from several languages.

Keywords: Direct object Marking. Affectation. Bantu language. Kimwani.

Resumen: Describimos y analizamos el patrón de marcación de los objetos directos en Kimwani. Describimos la tipología de DOM y presentamos evidencias de que la DOM no está relacionada con la escala o el grado de afectación, sino por la jerarquía de relevancia del objeto: los rasgos [+ humano] y [+ animalidad] están en el punto más alto de la jerarquía, y requieren marcación diferencial en la posición de objeto directo. Este resultado apunta a una dirección contraria de la propuesta teórica de Dowty (1991) con datos del inglés, Hopper y Thompson (1980) y Næss (2004) con datos de varios idiomas.

Palabras clave: Marcación del objeto directo. Afectación. Lengua bantu. Kimwani.

\footnotetext{
* Doutorando do Programa de Pós-Graduação em Linguística da Universidade Federal de Santa Catarina e docente da Universidade pedagógica/Rovuma de Moçambique afete no Departamento de Ciências de linguagem, Comunicação e Artes. http://orcid.org/0000-0003-4748-1828

* Professor do Departamento de Literatura e Línguas vernáculas da Universidade Federal de Santa Catarina (UFSC), professor permanente do Programa de Pós-Graduação em Linguística da UFSC e Bolsista PQ-2 do CNPq. http://orcid.org/oooo-0002-3999-3893
}

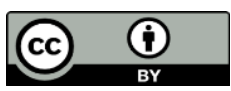

Este artigo está licenciado sob forma de uma licença Creative Commons Atribuição 4.0 Internacional, que permite uso irrestrito, distribuição e reprodução em qualquer meio, desde que a publicação original seja corretamente citada. 


\section{Introdução}

Antes de mais, precisamos retomar brevemente a noção de marcação em linguística que será importante para a análise que propomos neste artigo. Até onde se sabe, a abordagem da marcação em linguística foi, primeiramente, introduzida por fonologistas da escola de Praga no início do século XX (cf. TRUBETSKOY, 1939). Na segunda metade desse século, a discussão é retomada na fonologia por Chosmky e Halle (1968) e, em sintaxe, a marcação de oposições sintáticas e semânticas foi iniciada por Jakobson (1932). Segundo Comrie (2001 [1976]), em linguística, a intuição da noção de marcação pode ser definida nos seguintes termos:

quando há uma oposição entre dois ou mais associados (exemplo, perfectivo vs. imperfectivo), frequentemente este é o caso em que um dos associados da oposição é tido como sendo mais usual, mais normal, menos específico em relação ao outro. Em terminologias de marcação, o primeiro é não marcado e o outro é marcado (COMRIE, 2001 [1976], p.111. Tradução nossa').

Associado à noção de marcação, lembremos que todas as línguas naturais têm um padrão de funcionamento em que ocorrem orações transitivas e intransitivas as quais funcionam a partir de três categorias universais sintático-semânticas: Sujeito (S), Agente (A) e Objeto (O) (cf. DIXON, 1984; ONISHI, 20o1; AIKHENVALD 2001). Na língua bantu Mwani (doravante Kimwani), há um grupo de objetos diretos com traços [+humano] e [+animal] que é marcado em orações transitivas, diferentemente dos outros objetos diretos com traços [-animal] e [-humano] também em orações trasintivivas, como iremos demosntrar mais adiante com mais vagar.

Nesse contexto, o objetivo primeiro deste artigo é apresentar uma descrição e análise da Marcação Diferencial do Objeto direto (daqui em diante DOM, do original differential object marking, conforme LAZARD, 2001) em Kimwani, associando a questões nucleares da interface entre sintaxe e semântica. Mais especificamente, identificaremos e descreveremos a(s) tipologia(s) de marcação do objeto direto na

\footnotetext{
${ }^{1}$ Do original: "Where we have an opposition with two or more members (e.g. perfective versus imperfective), it is often the case that one member of the opposition is felt to be more usual, more normal, less specific than the other (in markedness terminology, it is unmarked, the others are marked)" (COMRIE, 2001 [1976], p.111). As traduções apresentadas neste artigo são de nossa responsabilidade.
} 
sentença nessa língua, considerando que não há uma descrição ainda realizada das interessantes propriedades da língua em tela.

Kimwani é uma das línguas bantu faladas no norte de Moçambique. E de acordo com a classificação de Guthrie (1967) apud Ngunga (2004, p. 46), das línguas Moçambicanas o Kimwani pertence a G: 45. Conforme Ngunga (2004), sobre a classficação das línguas bantu, Guthrie (1967-71) faz uma classificação geográficagenealógica das línguas, agrupando-as em 15 zonas codificadas por letras maiusculas, a saber, A, B, C, D, E, D, F, G, H, K, L, M, N, P, R, S. Internamente, cada zona divide-se em vários grupos de línguas, estabelecidos conforme critérios de proximidade ou distanciamento linguísitico e geográfico reflectindo um certo grau de proximidade genealógica. Cada grupo de línguas é codificado por um número decimal sufixado à letra do código da respectiva zona. Por exemplo: G.40, grupo Swahili, abrange as línguas Kiswahili, Kimwani, Cimakwe, etc. As línguas que constituem cada grupo são, por sua vez, codificadas através de unidades dentro desse número decimal. Por exemplo, a língua Kimwani tem o código 5 dentro do grupo 40, da zona G. Assim, G.45 significa, na classificação de Guthrie, língua Kimwani do grupo Swahili da zona G.

Conforme o mapa a seguir, Kimwani é falada na zona costeira da Província de Cabo Delgado/Moçambique, mais especificamente nos distritos de Ibo, Palma, Quissanga, Mocimboa da Praia e Pemba Cidade. De acordo com os dados de Instituto Nacional de Estatísitca - INE (2019), em 2017, data do último censo populacional, Moçambique possuía uma população de $27.909 \cdot 798$, sendo que (33.4\%) da população estava na zona urbana e (66.6\%) na zona rural. E de acordo com essa mesma fonte havia cerca de 77.915 falantes de Kimwani de cinco ou mais anos de idade. 
Mapa 1: Localização geográfica da região em Cabo Delgado/Moçambique onde Kimwani é falada em Moçambique

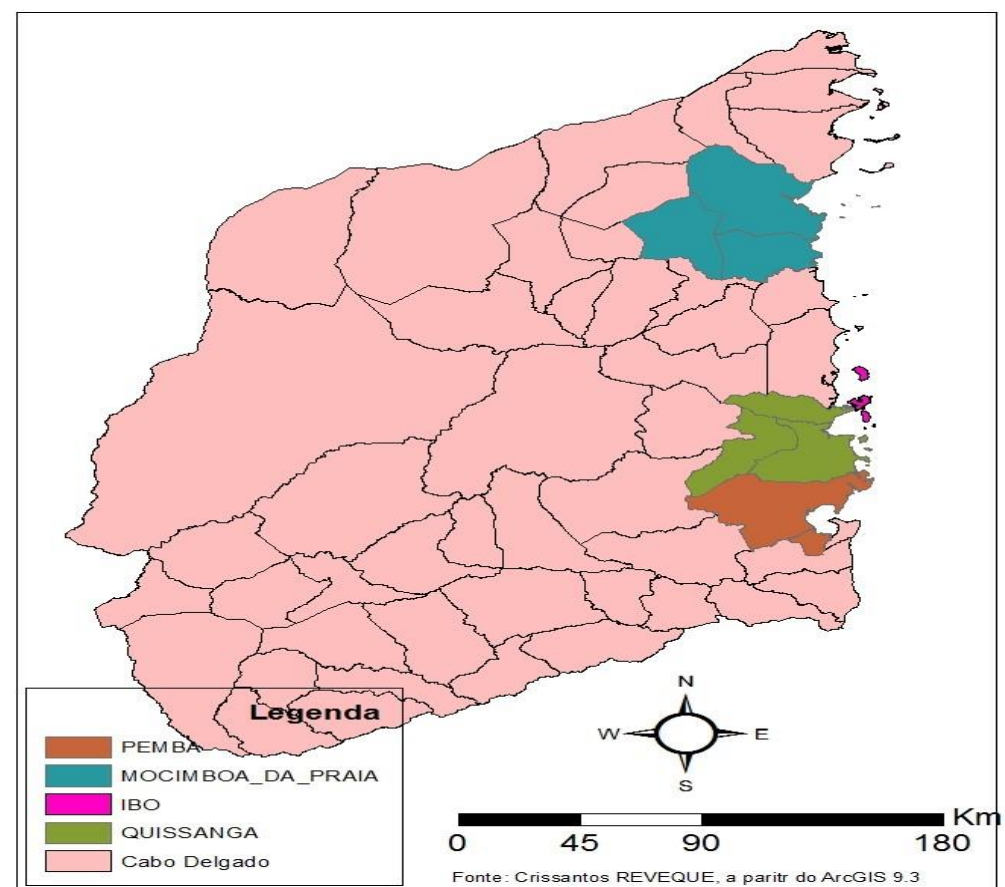

Fonte: departamento de ciências de terra e ambiente-Universidade Rovuma de Moçambique

Etimologicamente, a palavra Kimwani pode ser dividida em duas partes: $k i$ - é um prefixo nominal que significa moda ou modo, maneira; e mwani- é um tema nominal que significa praia, costa, litoral. Nisso, Kimwani quer dizer moda ou maneira da praia, moda da costa ou moda do litoral. Por extensão semântica, Kimwani também significa hábito, costume, modus vivendi do litoral.

O artigo está assim organizado em quatro seções. Em 1, apresentamos os procedimentos metodológicos e os pressupostos teóricos utilizados para a recolha, categorização e análise interpretativa dos dados. Em 2, exibimos algumas propriedades morfossintáticas e semânticas do Kimwani, especificamente, a marca de objeto em Kimwani. Em 3, descrevemos e analisamos dados de Marcação Diferencial do Objeto direto em Kimwani e, em 4, apresentamos as considerações finais seguidas das referências.

\section{Procedimentos metodológicos e pressupostos teóricos}


Os dados do Kimwani analisados e citados neste artigo fazem parte da base de dados da Dissertação de Mestrado de Salimo (2016), coletados em 2015 no distrito de Quissanga, província de Cabo Delgado/Moçambique. Quase cinco anos depois, esses dados são reanalisados qualitativamente neste artigo, a fim de explicar as tipologias e propriedades de legibilidade para a Marcação Diferencial do Objeto direto. A questão norteadora que nos colocamos aqui é: quais as propriedades de Marcação Diferencial do Objeto (DOM) direto em Kimwani? Para os propósitos deste artigo, não pretendemos entrar em pormenores teóricos, mas sim, com base em dados em uso da língua, descrever e explicar os mecanismos e as propriedades da DOM em Kimwani.

Como pressupostos teóricos, discutimos a proposta de Næss (2004) sobre a Marcação Diferencial do Objeto direto, em uma versão reinterpretada, a qual considera a animacidade e a definitude como conceitos relevantes para medir a afetação do objeto. De acordo com Næss (2004), há vários pontos sobre a Marcação Diferencial de Objeto que merecem destaques: primeiro, essa abordagem está em conflito com a noção difundida e estabilizada de transitividade, a qual considera o objeto típico como sendo altamente afetado, desde que a afetação possa ser demonstrada pela correlação ao alto grau de individuação. Segundo, a noção de marcação reversiva, empregada como um meio de fornecer uma descrição unificada de Marcação Diferencial de sujeito e objeto (DOM), não pode ser aplicada sem problemas com os tipos de oposições tipicamente envolvidas na DOM. Terceiro e último, as predicações feitas somente para a marcação de sujeito correspondem parcialmente aos dados linguísticos testados. Portanto, com base nestas constatações, Næss (2004) propõe uma perspectiva alternativa, uma análise que considera a marcação do caso acusativo como sendo a marca de alto grau de afetação do objeto, e explora a associação entre a afetação e o alto grau de individuação (definitude/animacidade).

Para Næss (2004), em uma oração transitiva o objeto que recebe o traço acusativo para além de ser altamente afetado deve ser também definido. Portanto, nesses casos o objeto é marcado. Em contrapartida, em orações transitivas em que o objeto não recebe o traço acusativo, tal objeto caracteriza-se por ser [-definido] e com um grau menor de afetação, consequentemente não marcado. 
Na visão de Næss (2004), tal análise pode explicar os dados de DOM, evitando assim as dificuldades inerentes às abordagens que levam os objetos individuados a serem semanticamente marcados.

Com vista a alcançarmos os objetivos propostos neste artigo, adotamos também a visão segundo a qual a realização das sentenças transitivas depende do número conceitual, semântico, e de propriedades morfossintáticas do evento e dos seus participantes (cf. DOWTY, 1991; HOPPER; THOMPSON, 1980), de modo que abordamos a marcação diferencial do objeto (DOM) olhando também para a individuação e afetação por serem propriedades que implicam as construções com objeto realizado.

A nossa abordagem é resultado de combinação de três perspectivas relacionadas. Primeiro, para a descrição e caraterização dos objetos recorremos às noções de afetação e individuação apresentadas por Hopper e Thompson (1980), e Dowty (1989). Segundo, para classificação do objeto como sendo marcado ou não marcado levamos em consideração os traços [definitude], [animal] e [humano] conforme Næss (2004). E a fim de nos permitir uma abordagem precisa e específica sobre a marcação do objeto em Kimwani, recorremos especificamente às orações transitivas da língua bantu em tela.

\section{Algumas propriedades morfossintáticas e semânticas do Kimwani}

O padrão de marcação de objeto nas diferentes línguas bantu é muito diverso, mas há muitos aspectos em comum entre si. Por exemplo, as línguas bantu são acusativas, e o Kimwani, particularmente, tem a marcação morfológica do argumento acusativo. As línguas bantu também mostram um padrão de concordância verbal categórica em número e pessoa entre Agente/Sujeito, como pode ser visto nos exemplos em Kimwani a seguir ${ }^{2}$.

(1) Juma ka-ebiya ngoma.

\footnotetext{
${ }^{2}$ Como já dito, todos os exemplos citados fazem parte da base de dados de Salimo (2016). Para compreender as abreviações usadas nos exemplos, fizemos uma pequena glosa: ${ }_{3} S G-3^{\underline{a}}$ Pessoa do singular; Ac - acusativo; MO - Marca de Objeto; MS - Marca de Sujeito; MT- Marca de Tempo; Nom.nominativo; Pas - Passado; VF - Vogal Final.
} 
Juma 3 SG-Pas-tocar tambor.

"O Juma tocou o tambor".

Em construções de duplo objeto sem preposição, o tema ocorre na posição adjacente posposta ao verbo e em seguida ocorre o objetivo/instrumento.

(2) Juma ka- jengera nyumba luwani. Juma 3SG-Pas-construir- casa - quintal.

"O Juma construiu quintal para a casa".

Em relação à atribuição de caso, em algumas orações, o sujeito em posição préverbal recebe o caso nominativo e desencadeia concordância com a forma verbal; o objeto recebe caso acusativo, e a concordância com o verbo não é categórica como se pode ver na sentença (3) abaixo apresentada. Nela, a forma verbal ka-Ø-jenga 'construiu' não recebe uma marca morfológica referente ao objeto nyumba 'casa'. O sujeito precede o verbo e o objeto está posposto (cf. HASPELMATH, 1984, p. 54-56).

(3) Juma ka- Ø-jenga nyumba.

Juma-Nom- 3SG-Ø-Pas-construir- casa-Ac.

"O Juma construiu uma casa".

Hopper e Thompson (1980, p. 251) afirmam que "a transitividade consiste num conjunto de dez (10) parâmetros semânticos" indicados em (4). Isto porque, para os autores, a "transitividade é uma caraterística da sentença e não da palavra, tal que uma atividade é transferida do agente para o paciente. A transitividade envolve um número de componentes, em que um deles é a presença do objeto do verbo". (HOPPER e THOMPSON, 1980, p. 251. Tradução nossa33).

\footnotetext{
3Do original: "Transitivity is traditionally understood as a global property of an entire clause, such that na activity is 'carried-over' or 'transferred' from an agente to a patient... We have identified the following parameters of Transitivity, each of which suggest a scale according to wihich clauses can be" (HOPPER e THOMPSON, 1980, p. 251).
} 
Quadro 1: Parâmetros semânticos da transitividade

\begin{tabular}{|lllc|}
\hline & & Alto & Baixo \\
A. & Participants & 2 or more participants, A and O & \\
B. & Kinesis & Action & No- action \\
C. & Aspect & Telic & Atelic \\
D. & Ponctuality & Puntual & Non-punctual \\
E. & Volitionality & Volitional & Non-volitional \\
F. & Affirmation & Affirmative & Negative \\
G. & Mode & Realis & Irrealis \\
H. & Agency & A high in potency & A low in potency \\
I. & Affectedness of O & O totally affected & O not affected \\
j. & Individuation of O & O highly individuated & O non-individuated \\
& & & \\
\hline
\end{tabular}

Fonte: Hopper e Thompson (1980, p. 251)

Estes dez parâmetros semânticos de transitividade permitem mostrar, de forma clara, que os componentes de transitividade na sentença envolvem diferentes perspectivas de eficácia e de intensidade com as quais a ação é transferida de um participante para o outro e tais componentes co-variam de língua para língua. Com base nestes parâmetros, as sentenças com maior presença das características mais à direta seriam mais transitivas do que as sentenças com características à esquerda.

Neste artigo, tomamos alguns desses parâmetros como indispensáveis para a caracterização dos objetos em orações transitivas do Kimwani. Com base nos nossos propósitos, sobre análise das propriedades que influenciam na Marcação Diferencial do Objeto, recorremos, mais especificamente, como relevantes as noções de individuação/definitude, afetação e número de participantes. Em relação a este último parâmetro, consideramos orações com dois participantes, Agente e Objeto.

Passemos, na seção seguinte, à descrição e à análise qualitativa dos dados.

\section{Marcação de Objeto Direto em Kimwani}

No campo de marcação, de acordo com Jakobson (1932, p. 74), "marcado é quando um membro de uma correlação deve conter um recurso ausente no membro não marcado". 
Morfologicamente, a marca do objeto em Kimwani realiza-se de várias formas: no singular, usam-se as formas $m u-, m w-, m o-, m w, m-, n-;$ no plural, as formas $w(a), w(e)$, $w(o), w(i), w(u)$ ou por meio de pronomes pessoais. Em princípio, semanticamente, das 18 classes nominais do Kimwani, a classe 1 (cl.1) é reservada aos seres humanos, essas marcas indicam de forma geral o traço [+animal]. A realização ou omissão do objeto na sentença em Kimwani depende do tipo de construção e associação semântica, de modo que o DP (Determiner Phrase) pode ocorrer de quatro modos: (a) sem marca de objeto; (b) com marca de objeto (MO) no verbo; (c) com marca do objeto (MO) no verbo, mas sem a realização plena do DP, isto é, com o DP objeto interno omisso; e, finalmente, (d) sem DP e sem a presença da marca do objeto no verbo na sentença. Seguindo notações de Ngonyani (1998, p.73), a realização do objeto em Kimwani pode ser sistematizada de seguinte forma:
a. $\varnothing-$ verbo DP
b. MO - verbo DP
c. $\mathrm{MO}$ - verbo $\varnothing$
d. $\varnothing$ - verbo $\varnothing$

Com base nesta notação, DP indica a presença do objeto lexical, MO indica a presença da marca do objeto e $\varnothing$ indica ausência tanto do objeto lexical assim como da marca do objeto. Essas categorias morfológicas foram aplicadas numa análise qualitativa em nossos dados, conforme os exemplos abaixo.

a. Nfalume ka-sower- a na mwivi. cl.1- Autoridade 3 SG-Pas-falar-VF com cl.1-ladrão 'Autoridade falou com o ladrão.'

b. Nfalume ka-m-sower- a mwivi. cl.1- Autoridade 3SG- MO-cl.1Pas-falar-VF cl.1-ladrão 'Autoridade falou sobre/do o ladrão.'

c. Nfalume ka- m- $\underline{\text { sower- }}$ a $\emptyset$ cl.1- Autoridade 3SG MO-cl. Pas-falar-VF 'Autoridade falou sobre/de ele.'

d. Nfalume ka- sower- $^{-} \mathrm{a}$ na mwivi? cl.1-Autoridade $3 S G$-Pas-falar-VF com cl.1-ladrão 
'A autoridade falou com o ladrão?

Aye, ka-sower-a

'Sim, ela falou.'

Em (6a) temos uma sentença sem a marcação diferencial do objeto direto, mas com o objeto [mwivi] lexicalizado. Ao passo que em (6b) temos uma construção em que, morfologicamente, ocorre um material linguístico extra, o morfema ' $m$-' como marca de objeto (MO) no verbo referente ao objeto interno, mwivi 'ladrão'. Diferentemente na sentença (6c) em que apesar de também ocorrer a marca morfológica de objeto (MO) no verbo, a MO não corre lexicalmente no objeto direto. Portanto, a marca do objeto (MO) tem a função pronominal, e a identificação do objeto é baseada nos seus traços/nas suas caraterísticas, tais como a classe nominal e o número pelo qual foi codificado a marca do objeto, neste caso em cl.1. De acordo com Onishi (2001) nas línguas de marcação de núcleo "as funções sintáticas são mostradas por elementos pronominais vinculados ao verbo" (ONISHI, 2001, p. 6. Tradução nossa ${ }^{4}$ ). Assim, por exemplo, a função sintática do argumento nuclear pode ser identificada ou por marcas de caso ou por marcas verbais. Em (6d) temos uma pergunta e uma resposta. Esta última trata-se de uma construção do tipo sentença elíptica na qual não ocorre nem a marca do objeto (MO) no verbo e nem o DP objeto interno. Nesta sentença, apesar do paciente não estar expresso, ele é, necessariamente, entendido como existente.

Dessa forma, os nossos dados mostram que a marcação do objeto em Kimwani é determinada por três fatores, sendo dois semânticos: definitude versus especificidade, e animacidade e um sintático função pronominal conforme Ngonyani (1988) e Hyman e Morolong (1977).

De acordo com Hopper e Thompson (1980) "afetação do objeto é o grau em que uma ação é transferida para um paciente é uma função de quão completamente esse paciente é afetado" (HOPPER e THOMPSON, 1980, p. 252-253. Tradução nossa5). Isso pode ser visto no exemplo (7), em Kimwani:

\footnotetext{
${ }^{4}$ Do original: "In head-marking languages syntactic functions are shown by bound pronominal elements on the verb" (ONISHI, 2001, p. 6).

${ }^{5}$ "Affectedness of O: the degree to wich na action is tranferred to a patiente is a function of how completely tha patient is affected" (HOPPER E THOMPSON, 1980, p. 252- 253).
} 
(7) (a) Omi nidangula nyumba 'eu destruí a casa'.

(b) Omi nidangula kipande ca nyumba 'eu destruí parte da casa'.

No primeiro caso, em (7a), a afetação é total, pois, pressupõe que o objeto nyumba (casa) foi totalmente destruída. Ao passo que, no segundo caso, em (7b), foi uma destruição parcial, kipande ca nyumba, parte da casa.

Em relação à noção de objeto definido ou objeto individuado, referimos tanto à distinção entre o Paciente e Agente assim como à distinção dos objetos em relação à presença ou não de todas as propriedades de substantivos apresentados no Quadro 2 a seguir. Para sustentar essa distinção, Hopper e Thompson (1980) apresentam um par de propriedades em que os nomes referentes aos substantivos mais à esquerda são mais definidos e os da direita menos definidos.

Quadro 2: Par de propriedades dos substantivos

\begin{tabular}{|ll|}
\hline $\begin{array}{l}\text { Individuado } \\
\text { Próprio }\end{array}$ & $\begin{array}{l}\text { Não individuado } \\
\text { comum }\end{array}$ \\
Humano, animal & inanimado \\
Concreto & abstrato \\
Singular & plural \\
Contáveis & massa \\
\hline
\end{tabular}

Fonte: Hopper e Thompson (1980, p. 253)

De acordo com Oslen e Resnik (1997), a "individuação do objeto do agente contrasta a baixa transitividade de objetos reflexivos com objetos de alta transitividade distinguidos claramente pela presença do agente" (OSLEN e RESNIK 1997, p. 330. Tradução nossa ${ }^{6}$ ). A individuação do objeto por ele próprio refere-se à habilidade de distinção do objeto direto por outros do mesmo tipo ou classe, na transitividade desses objetos, o peso assim como a altura, entre outros (tais como nome próprio versus nome

${ }^{6}$ Do original: "Individuation of the object from the agent contrasts low transitivity reflexive objects with high transitivity objects clearly distinguished from the agente" (OSLEN e RESNIK 1997, p. 330). 
comum), e o objeto referencial (definido versus indefinido) e não referencial, conforme Oslen e Resnik (1997).

Partindo do pressuposto de que os nomes das línguas bantu estão semanticamente agrupados em classes e aos pares, que variam de 18 a 20 (cf. BLEEK, 1862; MEINHOF, 1932; GUTHRIE, 1967), esperávamos que a animacidade e a definitude fossem condições de legibilidade para a Marcação Diferencial do Objeto direto em Kimwani, devido ao alto grau de afetação. No entanto, como mostramos com a análise dos dados acima, a marcação diferencial no objeto direto (DOM) nessa língua não parece estar relacionada com a escala ou grau de afetação. O nosso argumento é de que o DOM é requerido pela hierarquia de relevância do objeto e, nisso, os traços [humano] e [animalidade] estão no ponto mais alto da hierarquia, o que se requer marcação diferencial.

Sobre este aspecto, Morolong e Hyman (1977, p. 202), a fim de mostrarem que a marca do objeto é determinada pela posição do objeto na hierarquia de animalidade nas línguas naturais, afirmam que

quanto à animalidade um argumento é referenciado como alto com base na seguinte hierarquia de pessoa: $1^{\underline{\underline{a}}}>2^{\underline{a}}>3^{\underline{a}}$ humano $>3^{\underline{a}}$ animal $>3^{\underline{a}}$ inanimal, [...] tende a ter mais propriedades de objeto direto do que aqueles argumentos que tem uma referência baixa nesta escala/hierarquia (MOROLONG e HYMAN, 1977, p. 202. Tradução nossa7).

No entanto, para Levin e Rappaport (2005 p. 154),

as duas maiores concepções linguísticas sobre a proeminência apelam à hierarquia temática. Primeiro, o ranqueamento dos papeis temáticos é determinado por propriedades estruturais da representação da semântica lexical - as propriedades são definidas como a decomposição do predicado ou estrutura do evento. Segundo, o ranqueamento é determinado por vinculações associadas com o argumento (LEVIN e RAPPAPORT, 2005, p. 154. Tradução nossa ${ }^{8}$.

\footnotetext{
7“Animacy: An argument whose referent is higher in the following personal hierarchy, ist> 2nd> 3rd human $>3$ rd animal $>$ rrd inanimate [incomplete], will tend to have more DO properties than an argument whose referent is lower in this hierarchy" (MOROLONG e HYMAN, 1977, p. 202).

8 "We show that two major conceptions of prominence find their way into analyses of linguistic phenomena that appeal to a thematic hierarchy. On the first, the ranking of semantic roles is determined by structural properties of a lexical semantic representation - properties defined over a predicate decomposition or event structure. On the second, the ranking is determined by entailments associated with arguments" (LEVIN e RAPPAPORT, 2005, p. 154).
} 
Para esses autores, as duas concepções suportam a ideia central de que o mapeamento da semântica lexical para a sintaxe, em ambas as vias, precisa fazer referência aos argumentos na estrutura. Um dos exemplos de ranqueamento dos papéis semânticos dos argumentos apresentados pelos autores, extraídos em (BRESNAN; KANERVA, 1989; GIVÓN, 1984b, 2001; KIPARKY, 1985; GRIMSHAW, 1990) são de “Agente>instrumento>Paciente/Tema” (cf. LEVIN; HOVAV, 2005, p.156).

De acordo com Haspelmath (1984), existe uma larga variedade de condições das quais desviam o esquema de marcação do sujeito e do objeto. Nas palavras do autor, "tais variedades podem ser divididas em três classes: (i) condições relacionadas à referência, (ii) condições relacionadas à oração, e (iii) condições relacionadas ao predicado" (HASPELMATH, 1984, p. 56. Tradução nossa9). Em cada caso, os fatores envolvidos podem ser relacionados a um dos parâmetros de transitividade de Hopper e Thompson (1980). Os desvios na marcação canônica do argumento ocorrem se a transitividade é particularmente alta ou baixa. Ainda sobre a variação no padrão de marcação de DPs, Onishi (2001) explica que existem variações nos padrões canônicos de marcação de casos. Afirma a autora que "os DPs Agente e Objetos que levariam casos canonicamente podem ser marcados de forma diferente principalmente quando toda a oração exibe um baixo status de transitividade" (ONISHI, 2001, p. 5. Tradução nossa ${ }^{10}$ ), no sentido proposto por Hopper e Thompson (1980). Isto pode ocorrer, principalmente, quando: o status referencial de tais DPs é [não específico, indefinido, inanimado etc.] e/ou devido ao tipo de oração geral [na polaridade negativa, no aspecto imperfectivo, no irrealis, na estativa, no genérico, etc], conforme Onishi (2001).

Ainda na perspectiva de Haspelmath (1984), uma das condições relacionadas à referência é o alto grau de individuação/definição do objeto ou sua posição alta na hierarquia da animacidade e definitude. Nestas circunstâncias, muitas línguas mostram casos especiais na marcação de objeto (para distingui-los claramente do sujeito). Conforme Comrie (1989), até mesmo quando o objeto está em outras formas ele é

\footnotetext{
9"These can be divided into three classes: (i) reference-related condition, (ii) clause-related condition, and (iii) predicate-related conditions" (HASPELMATH, 1984, p. 56).

10"There are also variations in canonically marked case patterns. A/O/S NPs, which otherwise would take canonically marked cases, could be marked differently when the whole cluuse shows low transitivity status" (ONISHI 2001, p. 5).
} 
completamente não marcado e esse tipo de marcação não canônica é chamado de marcação diferencial do objeto (cf. HASPELMATH, 1984).

Vejamos alguns de nossos exemplos em Kimwani a seguir.

(9) ka- $\boldsymbol{n}$ - $\underline{\text { tafun- }}$ a ãngisi. 3SG-MO-Pas-comer-VF lula.

'Ele comeu uma/parte da lula'.

(10) ka - Ø-tatun- a ãngisi. 3SG- Ø -Pas-comer-VF lulas.

'Ele comeu as lulas'.

(11) a. Juma ka- $\boldsymbol{m}$-mon- a muka mwema. Juma 3 SG- MO-Pas-ver-VF mulher bonita. 'Juma viu uma mulher bonita'.

b. Juma ka- $\varnothing$-vunj- a bisikeleta. Juma 3 SG - Ø -Pas-quebrar-VF bicicleta. 'Juma quebrou a bicicleta'.

As sentenças apresentadas em (9), (10) e (11) mostram a ocorrência da marcação diferencial do objeto direto em Kimwani (DOM). O material morfológico extra que indica a marcação do objeto ocorre no interior da forma verbal, como em (9) e (11a), e, quando presente, é referenciado pela MO e $\varnothing$ pela ausência. O objeto direto das sentenças (10) e (11b) não são marcados, por isso, a forma verbal ocorre sem a presença da marca de objeto $\varnothing$. Isto é problemático para teorias que consideram a correlação entre o grau de afetação e a marcação. Neste caso concreto, em (10) e (11b) o grau de afetação dos objetos é total.

A seguir apresentamos dados que mostram os padrões que exigem na aplicação da marcação diferencial dos objetos diretos na língua em estudo.

(12) (a) Ka- Ø- dangul- a ngalawa.

3SG - Ø-Pas-destruir-VF barco.

'(Ele) destruiu o barco'.

(b) Ka - $\varnothing$-dangul- a mangalawa. ${ }_{3} \mathrm{SG}$ - Ø-Pas-destruir-VF barcos.

'(ele) destruiu os barcos'.

(c) Juma ka - Ø- jeng- a luwani. 
Juma $3 S G-\emptyset$-Pas-construir-VF quintal.

'O Juma construiu o quintal'.

(d) Juma ka- $\emptyset$-jeng- a maluwani. Juma $3 S G-\emptyset$-Pas-construir-VF os quintais.

'O Juma construiu os quintais'.

(e) Awa ke- Ø-fy- a riwe.

Awa 3 SG-Ø-Pas-atirar-VF pedra.

'Awa atirou uma pedra'.

(f) Awa ke- $\varnothing$-fy- a mawe.

Awa $3 \mathrm{SG}$ - $\emptyset$-Pas-atirar-VF pedras.

'Awa atirou pedras'.

Os dados em (12) são sentenças em que a afetação dos objetos é evidente e total. Analisando só a natureza dos DPs objetos internos diretos das sentenças, vemos que são todos de natureza [-animal]. E tanto nos casos [+definido] (12a, 12c, e 12e) assim como nos casos [-definido] (12b, 12d; 12f) não são sujeitos à aplicação da DOM. Portanto, em Kimwani em orações transitivas com o objeto direto não animal é geralmente não marcado mesmo que o objeto seja [+afetado] e [+ definido]. Os dados abaixo apresentados mostram um comportamento diferente:

(13) (a) ka- mu- lay- a mbuzi. 3SG -MO-Pas-matar-VF cabra.

'(ele) matou uma cabra'.

(b) Ko- Ø- lay- a mbuzi.

3SG - Ø -Pas-matar-VF cabras.

'(Ele) matou as cabras'.

(c) Juma ka -n - tafun- a ng'ombe. Juma 3SG-MO-Pas-comer-VF boi.

'O Juma comeu uma carne de boi'.

(d) Juma ka- $\varnothing$-tafun- a ng'ombe. Juma $3 \mathrm{SG}-\varnothing$-Pas-comer-VF boi

'O Juma comeu carne de boi' (não quantificada).

(e) Juma ka- $\boldsymbol{n}$ - mon- a nyuni. Juma 3 SG -MO-Pas-ver-VF pássaro.

'O Juma viu um pássaro'.

(f) Juma ka- $\varnothing$ - won- a nyuni. Juma $3 S G$ - $Ø$-Pas-ver-VF pássaros.

'O Juma viu os pássaros'. 
Em (13), temos sentenças em que os objetos diretos são semanticamente de natureza [+ animal]. Os objetos diretos das sentenças (13a, 13b, 13c e 13d) são completamente afetados pela ação do agente, mas a DOM só é exigida quando o DP objeto interno direto é [+definido/+ individuado], ou [+específico]. Ao passo que, nos casos em que o DP objeto direto é [-definido/-individuado] ou [-especifico] não ocorre a DOM, mesmo quando a afetação é completa (13b e 13d). Em (13e) e (13f), a forma verbal -won- 'ver' não acarreta uma afetação do objeto direto. Mas, mesmo não acarretando uma afetação do objeto direto, em (13e) esse é marcado diferencialmente com um material linguístico extra dentro da forma verbal ka- $n$ - mon-a 'viu-o', por ser [+ definido/+individuado] ou [+específico]. A única diferença entre (13e) e (13d) é a definitude/individuação dos seus objetos diretos. Vejamos em seguida dados em que os objetos internos direto são de natureza semântica [+ humano].

(14) (a) Juma ka-n - kol - a ntiri. Juma 3 SG -MO-Pas-pegar-VF fugitivo.

'O Juma pegou o fugitivo'.

(b) Juma ka-wa-kol- a wevi. Juma 3SG -MO-Pas-pegar-VF ladrões.

'O Juma pegou os ladrões'.

(c) Awa ka -n- sukul- a kisimana. Awa 3SG-MO-Pas-levar-VF criança. 'Awa levou a criança'.

(d) Awa ka - wa -sukul- a wasimana. Awa 3 SG -MO-Pas-levar-VF crianças. 'Awa levou as crianças'.

(e) Mwivi ka-n- $\underline{\text { lumiz- }}$ a munu. Mwivi 3SG -MO-Pas-aleijar-VF pessoa. 'O ladrão aleijou uma pessoa'.

(f) Mwivi ka - wa - lumiz- a wanu. Mwivi 3SG -MO-Pas-aleijar-VF pessoas.

'O ladrão aleijou as pessoas'.

As sentenças em (14) são semanticamente de natureza diversa, uma vez que, por um lado, ocorrem verbos que acarretam afetação parcial, como em (14a e 14 b) -kol'pegar' e ocorre uma marca morfológica (n-, m-) dentro da forma verbal referente ao 
objeto. Por outro, ocorrem verbos em que a ação do agente afeta completamente o paciente, como, por exemplo (14e e 14f) -lumiz- 'ferir' ou 'golpear' também ocorre um material linguístico extra (n-, m-) dentro da forma verbal.

As sentenças em (14) estão formadas parcialmente aos pares (a, b), (c, d) e (d, f). Em cada par de sentenças uma está no singular e a outra no plural. Em relação aos objetos direto, a diferença entre cada par está nos traços de definitude/individuação e de especificidade dos DPs objetos internos diretos. Quanto às semelhanças é importante referir que todos os objetos internos diretos presentes nas sentenças em (14) são semanticamente de natureza [+humano].

No que concerne à marcação, tanto nos casos com traços [+definido/+individuado] e [+específico], como em (14a, 14c e 14e), assim como nos casos com traços [-definido/-individuado] e [-específico], como em (14b,14d e14f), ocorre a marcação diferencial do objeto direto (DOM) por meio de um material linguístico extra (-n-, -wa-). Portanto, em Kimwani, a aplicação da DOM por meio de um morfema dentro da forma verbal é categórica quando o objeto direto é de natureza [humano], independentemente da afetação, definitude ou não do objeto.

Os resultados em (12), (13) e (14) confirmam o nosso argumento, segundo o qual, a marcação diferencial no objeto direto (DOM) em Kimwani não está relacionada com a escala ou grau de afetação, mas, sim, pela hierarquia de relevância do objeto na língua, de modo que traços como [+humano] e [+animado] estão no ponto mais alto da hierarquia, e requerem a marcação diferencial. Essa proposta de análise para o Kimwani é corroborada pela proposta de Morolong e Hyman (1977, p. 202), segundo a qual a marcação do objeto é determinada pela posição do objeto na hierarquia de animalidade. Esses autores afirmam que "um dos argumentos é referente altura na escala/hierarquia de pessoa, $1^{\underline{a}}>2^{\underline{a}}>3^{\underline{a}}$ humano $>3^{\underline{a}}$ animal $>3^{\underline{a}}$ não animal, [...] tende a ter mais propriedades de objeto direto do que aqueles argumentos que têm uma referência baixa nesta escala/hierarquia"11.

Os resultados que apontamos neste artigo com dados do Kimwani, portanto, sugerem que, a proposta de Næss (2004) sobre a marcação diferencial do objeto direto,

${ }^{11}$ Veja a citação de Haspelmath (1984, p. 56), transcrita na nota 11 neste artigo. 
a qual considera a animalidade e a definitude como conceitos relevantes para medir a afetação do objeto, deve ser relativizada. Os dados do Kimwani mostram que a animacidade e a definitude são relevantes na hierarquização dos argumentos, mas não para medir a afetação, de modo que, os argumentos com traços [+humano], [+ animal] e [+definido] exigem a marcação diferencial de forma categórica, independentemente do grau/escala da afetação.

\section{Considerações finais}

Os dados analisados neste artigo nos permitem afirmar que, para além de essa língua seguir o padrão de marcação do argumento acusativa, o Kimwani também exibe um padrão de concordância categórica em número e pessoa entre Agente/Sujeito e a forma verbal. A realização ou omissão do objeto direto (DP) depende do tipo de construção e associação semântica. E, nesse sentido, o DP pode ocorrer sem a marca do objeto (MO); o DP pode ocorrer com marca de objeto (MO) no verbo; pode ocorrer a marca do objeto no verbo, mas sem a realização plena do DP, isto é, com o DP objeto interno omisso; e, finalmente, pode ocorrer uma sentença sem DP e sem a presença da marca do objeto (MO) no verbo.

Os resultados parecem contrariar a hipótese de Næss (2004), pois mostram que a marcação diferencial no objeto direto (DOM) em Kimwani não está relacionada com a escala ou o grau de afetação. Os dados revelam que a DOM é requerida pela hierarquia de relevância do objeto na língua e, nisso, os traços [humano] e [animalidade] estão no ponto mais alto da hierarquia e requerem a marcação diferencial, visão defendida por Morolong e Hyman (1977) e Haspelmath (1984), os quais salientam que uma das condições relacionadas à referência é o alto grau de individuação/definição do objeto ou sua posição alta na hierarquia da animacidade e definitude.

Neste artigo, a fim de identificarmos as propriedades de marcação diferencial do objeto, focamos especificamente na natureza do objeto direto com recurso a orações transitivas e deixamos de fora as propriedades do DP com função de sujeito em Kimwani. Também não abordamos sentenças de duplo objeto. Para uma investigação futura seria 
importante não só uma abordagem associada aos dois elementos, mas também a inclusão de sentenças interrogativas e relativas.

\section{Referências}

AIKHENVALD, Alexandra Y. Verb types, non-canonically marked arguments and grammaticalrelations: ATariana perspective. 2001. In: ALEXANDRA Y. Aikhenvald R.M.W. Dixon \& MASAYUKI Onishi. (edits). Non-Canonical Marking of Subjects and Objects. John Benjamins Publishing Company. Vol. 45. Amsterdam/Philadelphia, 2001. p. 177-200.

BRESNAN, J \& KANERVA, JM. Locative inversion in Chichewa: A case study of factorization in grammar. Linguistic inquiry, JSTOR, 1989.

BLEEK, Wiherm. A Comparative Grammar of South African Languages. London: Trübner, 1862.

COMRIE, Bernard. Aspect. Cambridge:Univesity Press, 1976. . Language universals and linguistic typology. 2nd edn. Oxford:Blackwell, 1989.

CHOMSKY, N. and HALLE, M. The sound pattern of English. New York: Harper and Row, 1968.

DIXON, R.M.W. The semantic basis of syntactic properties. Proceedings of the Tenth Annual Meeting of the Berkeley Linguistics Society, 1984. p. 583-595.

DOWTY, David R. Thematic proto-roles and argument selection. Language 67, 1991. p. 547-619.

On the semantic content of the notion 'thematic role'. Properties, types and meanings, vol. II, ed. by PARTEE, Barbara, CHIERCHIA, Gennaro \& TURNER, Ray. Dordrecht: Kluwer, 1989. p. 69-130.

GIVÓN, T. Syntax: A Functional-Typological Introduction, vol. I, John Benjamins, Amsterdam, 1984b.

Syntax: An Introduction, vols. I and II, John Benjamins, Amsterdam. Second edition, 2001. 
GUTHRIE, Malcolm. Comparative Bantu: an introduction to the comparative linguistics and prehistory of the Bantu languages. 4 vols. Letchworth UK \& 276 Brookfield VT: Gregg International, 1967/71.

GRIMSHAM, J. Argument structure. Cambridge: MIT Press, 1990.

HASPELMATH, Martin. Transitivity alternations of the anticausative type. (Arbeitspapiere, N.F., Nr. 4) Cologne: Institut für Sprachwissenschaft der Universität zu Köln, 1984.

HYMAN, Larry \& MOROLONG, Malillo. Animacy, objects, and clitics in Sesotho. Studies in African Linguistics 8, 1977. p. 199-217.

HOPPER, P.J. \& THOMPSON, S. A. Transitivity in gramar and discourse. Language 56, 1980. p. 251-299.

JAKOBSON, R. Zur Struktur des russichen Verbums. In Charisteria. G. Mathesio. Prague: Cercle Linguistique de Prague, 1932. p. 74-84.

KIPARKY, P. Morphology and Grammatical Relations, unpublished ms., Stanford University, Stanford, CA, 1985.

LAZARD, G. Le marquage diférentiel de l'objet. 2001. In: Language typology and language universals: An international handbook, M. Haspelmath, E; König, W. Oesterreicher and W. Raible (edits.). Berlin: Mouton de Gruyter, 2001.

LEVIN, Beth and HOVAV, Malka Rappaport. Research Sueveys in Linguistics, Argument Realization. Cambridge University Press, 2005.

MEINHOF, Carl. Introduction to the Phonology of the Bantu Languages. Berlim: Dretrich Reimer, 1932.

NÆSS, Åshild. What markedness marks: the markedness problem with direct objects. Língua 114, 2004. p. 1186-1212. Disponível em:<www.sciencidirect.com>._Acesso em: 15 nov 2018.

NGONYANI, Deografia. Properties of Applied objects in Kiswahili and Kindendeule; Studies in African Linguistics, Volume 27, Number 1, spring, 1998.

NGUNGA, Armindo. Introdução à Linguística Bantu. Imprensa Universitária. UEM. Maputo, 2004.

ONISHI, Masayuki. Introduction: Non-canonically marked subjects and objects:Parameters and Properties. In: ALEXANDRA Y. Aikhenvald R.M.W. Dixon \& 
MASAYUKI Onishi. (edits). Non-Canonical Marking of Subjects and Objects. John Benjamins Publishing Company. Vol. 45. Amsterdam/Philadelphia, 2001. p. 1-52.

OLSEN, M.; RESNIK, P. Implicit Object Constructions and the (in)transitivity continuum 33rd Regional Meeting of the Chicago Linguistics Society, April, 1997. p. 327336.

Recenciamento Geral da População e Habitação de Moçambique. Instituto Nacional de Estatística (INE), Maputo, 2019. Disponível em: <www.ine.gov.mz>. Acesso em: 28 maio 2019 .

SALIMO, Calawia. Tom gramatical em Kimwani. Dissertação de Mestrado. Universidade Pedagógica de Moçambique, 2016.

TRUBETZKOY, N. S. Grundzuge der Phonologie. Travaux du Cercle Linguistique de Prague 7, 1939.

Recebido em 14/o1/2020. Aprovado em 18/04/2020. 